Türkiye Tarımsal Araştırmalar Dergisi
dergipark.gov.tr/tutad $\begin{aligned} & \text { Turk J Agric Res } \\ & \text { 2017, 4(1): 65-70 } \\ & \text { O TÜTAD } \\ & \text { ISSN: 2148-2306 } \\ & \text { e-ISSN: 2528-858X } \\ & \text { doi: 10.19159/tutad.300709 }\end{aligned}$

\title{
İyon-Seçici Sensörler Kullanılarak Bazı Sebzelerin Topraksız Bitki Yetiştirme Ortamlarındaki Nitrat, Potasyum ve Kalsiyum Tayini
}

\author{
Melda ALTIKATOĞLU ${ }^{1 *}$, İbrahim IŞILDAK ${ }^{2}$ \\ ${ }^{l}$ Ylldız Teknik Üniversitesi, Fen-Edebiyat Fakültesi, Kimya Bölümü, İstanbul, TÜRKiYE \\ ${ }^{2} Y$ ıldız Teknik Üniversitesi, Kimya-Metalurji Fakültesi, Biyomühendislik Bölümü, İstanbul, TÜRKIYE
}

\begin{abstract}
Geliş Tarihi/Received: 12.10 .2016
Kabul Tarihi/Accepted: 21.11 .2016

*Sorumlu Yazar/Corresponding author: maltikatoglu@yahoo.com

Özet: Bitkilerin arzuladığı minerallerin uzun süre kontrollü bir şekilde bitkiye aktarılması önem arz ettiğinden, basit ve ekonomik yeni mikro ve makro ölçekli tarım üretim sistemlerine ihtiyaç duyulmaktadır. Bu çalışmada, beslenme sürecinde bitki besin solüsyonu içindeki besin elementlerinin bitkinin ihtiyacına göre istenen düzeyde tutulması, bilgisayar kontrollü potansiyometrik mikro büyüklükte kimyasal sensör sistemler kullanılarak takip edilmiştir. Hidrofonik çözeltideki makro besin elementlerinden $\mathrm{NO}_{3}{ }^{-}, \mathrm{K}^{+}$ve $\mathrm{Ca}^{2+}$ iyonlarının derişimini hassas ve seçici olarak ölçmek için polivinilklorür (PVC) temelli iyon-seçici sensörler kullanılmıştır. Serada yetiştirilen yeşilbiber ve patlıcan fidelerinin ihtiyacı olan nitrat, potasyum ve kalsiyum iyon seviyelerinin farklı olduğu görülmüştür. Elde edilen sonuçlar, büyümenin ilk iki ayında, biber bitkisinin patlıcandan daha az miktarda nitrat tükettiğini göstermiştir. Ayrıca, bitkilerin tükettiği potasyum miktarının tayin edilen diğer besin elementlerinden daha fazla olduğu belirlenmiştir.
\end{abstract}

Anahtar Kelimeler: Topraksız tarım, besin elementleri, kimyasal sensör, hidrofonik sistem

\section{Determination of Nitrate, Potassium, and Calcium using Ion-Selective Sensors in Soilless Plant Growth Media of Some Vegetables}

\begin{abstract}
Since it is important to transfer the minerals desired by plants for a long time in a controlled manner, simple and economical new micro and macro scale agricultural production systems are needed. In this study, the nutrients in the plant nutrient solution were kept at the required level according to the needs of the plant, using computer controlled potentiometric micro-sized chemical sensor systems. Polyvinylchloride (PVC) -based ion-selective sensors have been used to sensitively and selectively measure the concentration of macro nutrient $\mathrm{NO}_{3}{ }^{-}, \mathrm{K}^{+}$, and $\mathrm{Ca}^{2+}$ ions in the hydroponic solution. It has been found that the nitrate, potassium and calcium ion levels required for the green pepper and eggplants grown in the greenhouse are different. The results showed that in the first two months of growth, the pepper consumed nitrate in a lesser amount than the eggplant. In addition, it was determined that the amount of potassium consumed by plants was higher than that of other nutrients.
\end{abstract}

Keywords: Soilless culture, nutrient elements, chemical biosensor, hydrophonic system

\section{Giriş}

Topraksız tarım, bitkilerin toprak kullanılmadan gereksinim duydukları organik ya da inorganik besin maddelerini kontrollü sera koşullarında yetiştirme ortamına verilerek yapılan üretim şeklidir. Toprakta üretimin yetersiz olduğu ya da mümkün olmadığı, iklim koşullarının uygun olduğu ortamlardan yararlanmak amacı ile topraksız tarıma ihtiyaç duyulmuştur (Benoit ve Ceustermans, 1995; Pardossi ve ark., 2004). Böylece tarım alanlarından daha fazla yararlanma imkânı doğmuştur. Topraksız tarım, su kültürü ve katı ortam kültürü olmak üzere iki şekilde yapılır. 
$\mathrm{Su}$ kültüründe, bitkiler su içerisinde gelişirken kontrollü olarak besin çözeltisi ile gübrelenir. Katı ortam kültüründe ise, perlit, torf, kaya yünü, pomza, çakıl, kum, gibi organik ve inorganik materyaller kullanılır (Kaplan ve ark., 1999; Raviv ve Lieth, 2008).

İlk kez 1930 yılında İngiltere'de Kaliforniya Üniversitesi'nden Profesör Doktor William Gericke tarafindan temelleri atılan bu yöntem, Hollanda tarafından geliştirildi. Türkiye'de ise ilk olarak 1995'te Antalya'da Agroser adlı serada kullanılmaya başlandı. Bugün topraksız tarım tekniği, Türkiye'de çoğu ihracat ağırlıklı üretim yapan yaklaşı 30 serada başarıyla uygulanmaktadır. İç pazara verdikleri ürün miktarı daha sınırlıdır. Çünkü bu teknikle üretilen ürünler daha pahalıdır. Topraksız tarımın amac1; bitkilerin gelişmesini besin solüsyonu ile sağlamak, bitkilerin besin madde ve su gereksinimlerini stres oluşturmadan karşılamakta ve bunu en ekonomik bir şekilde gerçekleştirmektir (Sönmez ve Kaplan, 2004; Raviv ve Lieth, 2008; Anonim, 2016).

Günümüzde topraksız tarımın seracılıkta hızla yayılmasının en büyük sebeplerinden birisi toprak kökenli sorunlardır (Jones, 2005). Topraksız kültür; toprak yorgunluğu, topraktan kaynaklanan hastalık ve zararlı gibi sorunların olmaması, gübre ve su ilişkisini denetleyerek bitki gelişimini kontrol altına almasi, topraktan kaynaklanan kaliteyi düşürücü unsurları ortadan kaldırması ve verimi arttırması gibi üstünlüklere sahiptir (Savvas, 2002; Meriç ve ark., 2011).

Topraksız tarımda kullanılan biyolojik testler tam garanti vermemekle birlikte maliyetli ve uzun süreli çalışmalardır. Gelişmekte olan üreticiler ticari koşullar altında bu kapsamlı testleri tercih etmemektedir. Yapılan laboratuvar testleri güvenilir sonuçlar vermeli, kısa süreli, düşük maliyetli ve referans örnekleri için standart koşullar sağlamalıdır (Blok ve ark., 2008). Herhangi bir besin elementinin miktarındaki değişiklikler bitki büyümesini etkileyebilmektedir. Bu yüzden, hidrofonik sistemde sensörler ile besin elementlerinin kontrolü, etkin bir bitki büyümesi için önemlidir (Gutierrez ve ark., 2007; Vardar ve ark., 2015). Hidrofonik makro besin elementlerinin miktarlarını hızlı ve sürekli ölçmek için iyon-seçici elektrot teknolojisine ihtiyaç duyulmaktadır (Bailey ve ark., 1988; Cloutier ve ark., 1997; Vardar ve ark., 2015).

$\mathrm{Bu}$ çalışmada; hidrofonik serada yetiştirilen yeşilbiber ve patlıcan bitkilerinin bitki besin çözeltisindeki nitrat, potasyum ve kalsiyum derişimleri potansiyometrik sensör sistemi ile doğrudan ve eş zamanlı olarak ölçülmüştür.
Bitkiler organik yetiştirme kuralları ile desteklenen bir bitki-besleme ortamı kurularak topraksız tarım tekniğiyle çalışan serada yetiştirilmiştir. Bitkinin ihtiyacı kadar kontrollü ve hılı bitki besin uygulaması ile daha kaliteli ve daha bol miktarda ürün elde edilebilir.

\section{Materyal ve Yöntem}

\subsection{Materyal}

$\mathrm{NO}_{3}{ }^{-}, \quad \mathrm{K}^{+}$ve $\mathrm{Ca}^{2+}$ için iyonoforlar, polivinilklorür (PVC), dioktilsebakat, potasyum tetrakisklorofenilborat, nitrofeniloktileter, tetradodesilamonyum nitrat, dibütilfitalat, tetrahidrofuran (THF), grafit, epoksi ve sensör hazırlamada kullanılan diğer tuzlar SigmaAldrich'den temin edilmiştir. Seradaki bitkilerin besin çözeltilerinin hazırlanması için makro ve mikro besin elementleri ticari formda farklı marketlerden alınmıştır. Bitki fideleri Türkiye'deki satış yerlerinden ticari olarak temin edilmiştir. Çalışma, Yıldız Teknik Üniversitesi'ne ait hidrofonik serada topraksız tarım koşullarında yürütülmüştür. Bitkilerin yetiştirildiği seranın resmi Şekil 1'de gösterilmiştir.

\subsection{Sensörlerin hazırlanması}

Bütünüyle katı hal PVC membran sensörler hazırlanırken, PVC membranlar metal yüzeyine iyi yapışmadığından hem membrana iyi tutunan, hem de metale kısmen tutunan özelliklere sahip bir ara yüzey (katı kontak) kullanılmıştır. Katı kontak materyal kütlece \% 50 grafit, \% 35 epoksi ve \% 15 sertleştiricinin yeterince THF içerisinde homojen olacak şekilde karıştırılıp, uygun kıvama gelene kadar THF'sinin uçurulması ve daha sonra metal yüzeyine kaplanıp kurumaya bırakılmasıyla hazırlanmıştır (Iş̧1dak ve ark., 2004; Çoldur ve ark., 2009). Elektrot yüzeyleri yukarıda bahsedilen katı kontak materyali ile kalınlığı yaklaşık $0.5 \mathrm{~mm}$ olacak şekilde kaplanıp kurutulmuştur. Daha sonra elektrod ilgili membran bileşimine (Tablo 1) yaklaşık 0.1-0.2 mm kalınlığında membran kalınlığı elde edilene kadar birkaç kere daldırılmıştır. Kaplanan membranlar 12 saat laboratuvar ortamında kurumaya birakıldıktan sonra $10^{-1} \mathrm{M}$ ana iyon çözeltisinde 6 saat süreyle şartlandırılmış ve elektrotlar ölçüm almaya hazır hale getirilmiştir.

Potansiyometrik ölçümler bilgisayar kontrollü çok kanallı potansiyometrik ölçüm sistemi ile oda sıcaklığında yapılmıştır. Ölçümlerde kullanılan çalışma elektrotları mikro boyutlu katı hal iyonseçici elektrotlardır. Referans elektrot olarak, mikro boyutlu katı gümüş/gümüş klorür elektrot kullanılmıştır. 


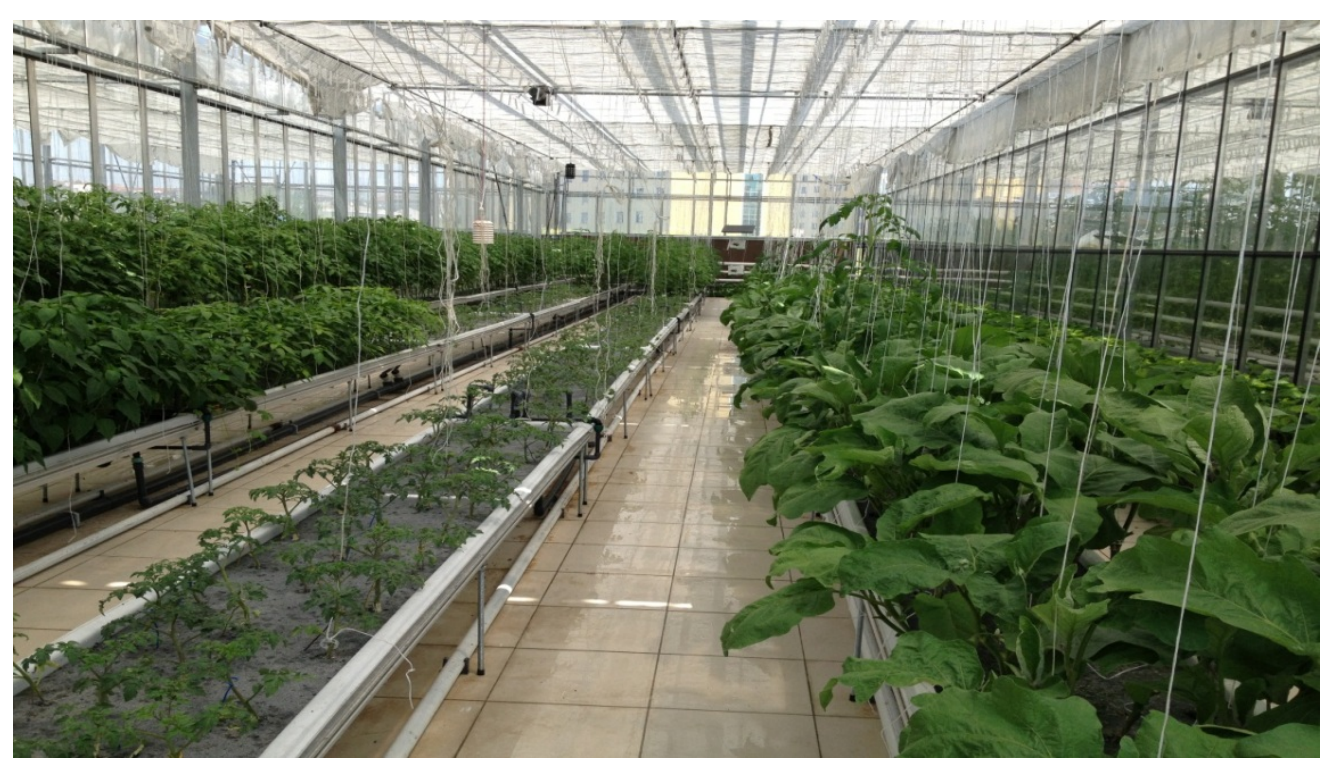

Şekil 1. Çalışma alanına ait biyoteknolojik sera

Tablo 1. Hazırlanan katyon ve anyon seçici sensörlerin membran bileşimler

\begin{tabular}{|c|c|c|c|c|}
\hline Sensör & İyonofor & Polimer & Plastikleştirici & İyonikliği artırıcı \\
\hline $\mathrm{K}$ & K İyonofor I (\% 1) & PVC (\% 33.5) & DOS (\% 64) & KTpClPB (\% 0.5) \\
\hline $\mathrm{Ca}^{2+}$ & Ca İyonofor IV (\% 2) & PVC (\% 29) & NPOE (\% 68) & KTpClPB (\% 1) \\
\hline $\mathrm{NO}_{3}^{-}$ & $\mathrm{TDA}^{-\mathrm{NO}_{3}(\% 4)}$ & PVC (\% 30) & DBF $(\% 65)$ & $\mathrm{KTpCIPB} \mathrm{( \%} \mathrm{1)}$ \\
\hline
\end{tabular}

Potasum, Ca: Kalsiyum, $\mathrm{NO}_{3}$ : Nitrat, TDA- $\mathrm{NO}_{3}$ : Tetra

\section{3. Çözeltilerin hazırlanması}

Elektrotların potansiyometrik olarak kalibrasyonu için iyonların deiyonize su ile hazırlanmış standart çözeltileri kullanılmıștır. Önce iyonların $10^{-1}$ M'lik standart stok çözeltileri hazırlanmıştır. Çözelti konsantrasyonlarında önemli bir hataya yol açmamak için düşük konsantrasyon değerindeki çözeltiler bu stok çözeltiden adım adım seyreltilerek ihtiyaç duyulan konsantrasyonlardaki çözeltiler hazırlanmıştır.

$\mathrm{K}^{+}$ve $\mathrm{NO}_{3}^{-}$elektrotlarının kalibrasyonu için $10^{-1}, 10^{-2}, 10^{-3}, 10^{-4}$ ve $10^{-5} \mathrm{M} \mathrm{KNO}_{3}$ çözeltisi; $\mathrm{Ca}^{2+}$ elektrodunun kalibrasyonu için ise $10^{-1}, 10^{-2}$, $10^{-3}, 10^{-4}$ ve $10^{-5} \mathrm{M} \mathrm{CaCl}_{2}$ çözeltisi hazırlanmıştır.

\section{Bulgular ve Tartışma}

\subsection{Hazırlanan elektrotların potansiyometrik ölçümleri}

Potasyum, nitrat ve kalsiyum için hazırlanan kat1-hal iyon seçici membran sensörlerin potansiyometrik performans özellikleri Tablo 2'de verilmiştir. Potansiyometrik performansları incelendiğinde tüm sensörlerin $0.1-100 \mathrm{mM}$ aralığında Nernstian cevap verdiği görülmektedir. Hazırlanan sensörlerin tayin limiti ve cevap süresi de oldukça iyidir. Gerçek örneklere elektrotların uygulamasında ortamda bulunabilecek değişik iyonlara karşı göstermiş olduğu seçiciliğin birer ifadesi olan seçicilik katsayıları (Kij) ayrı çözelti metoduna göre hesaplanmıştır (Tablo 2). Hazırlanan sensörlerin tekrarlanabilirliğini belirlemek için aynı günde aynı sensör ile 5 defa ölçüm alınmıştır ve bağıl standart sapmaları hesaplanmıştır. Sensörlerin bağıl standart sapma değerlerinin \% $\quad 1.0$ 'dan düşük bulunmasından dolayı, tekrarlanabilirliğin yüksek olduğu görülmüştür. Potansiyometrik duyarlılık, seçicilik, cevap zamanı, çalışma $\mathrm{pH}$ aralığı, tekrarlanabilirlik ve sensör ömrü açısından hazırlanan tüm sensörlerin hidrofonik seradaki besin çözeltilerinin tayininde kullanılabileceği öngörülmektedir.

\subsection{Bitki besin elementlerinin tayin edilmesi}

Hazırlanan elektrotların standart çözeltiler ile kalibrasyonu yapıldıktan sonra besin çözeltilerinin içerdiği potasyum, nitrat ve kalsiyum iyon konsantrasyonları hesaplanmıştır. $\mathrm{Bu}$ çalışmada kullanılan besin solüsyonları Yıldız Teknik Üniversitesi'nde bulunan "Biyoteknolojik Sera" dan temin edilmiştir. Seradaki bitki yataklarının giriş ve çıkış suları alınarak, bitki besin elementlerinin takibi bilgisayar kontrollü olarak 
mikro büyüklükte hazırlanan kimyasal sensörler kullanılarak sürekli ölçümler alınarak yapılmıştır. Serada yetiştirilen yeşilbiber ve patlıcan için bitkinin kullandığ 1 potasyum, nitrat ve kalsiyum miktarları hesaplanarak Tablo 3 'te gösterilmiştir.

Nitrat bitkinin büyümesi için gerekli olan önemli bir makro besin elementidir (Van Os ve ark., 2002). Elde edilen sonuçlara göre, bitkilerin büyüme sırasında tükettiği nitrat miktarı her geçen hafta değişiklik göstermiştir (Şekil 2). Yeşilbiber ve patlıcan için aynı miktarda nitrat kullanılmıştır. Şekil 2'de görüldüğü gibi, yeşilbiber patlıcandan daha az miktarda nitrat tüketmiştir. İlk iki hafta yeşilbiberin kullandığı nitrat miktarı aynı iken ikinci haftadan sonra az miktar artış gözlenmiştir. Bu durum, nitrat miktarındaki değişmenin bitkinin nitrat gereksinimine göre değiştiğini

Tablo 2. Potasyum, kalsiyum ve nitrat seçici sensörün performans özellikleri

\begin{tabular}{|c|c|c|c|}
\hline Belirlenen sensör özellikleri & $\mathrm{K}$ & $\mathrm{Ca}$ & Nitrat \\
\hline Konsantrasyon calısma aralığ & $10^{-5}-10^{-1} \mathrm{M}$ & $10^{-6}-10^{-1} \mathrm{M}$ & $10^{-6}-10^{-1} \mathrm{M}$ \\
\hline Tayin limiti & $4 \times 10^{-5} \mathrm{M}$ & $1 \times 10^{-6} \mathrm{M}$ & $5 \times 10^{-6} \mathrm{M}$ \\
\hline S1caklık çalışma aralığ & $5-50^{\circ} \mathrm{C}$ & $5-50^{\circ} \mathrm{C}$ & $5-50^{\circ} \mathrm{C}$ \\
\hline Membrane tip & Kompozit membran & Kompozit membran & $\begin{array}{l}\text { Kompozit } \\
\text { membran }\end{array}$ \\
\hline pH çalışma aralığ 1 & $5-9$ & $3-7$ & $2-7$ \\
\hline Seçiciliği & $\begin{array}{l}\log K_{K^{+}, B^{n+}}^{\text {pot }} \\
\mathrm{Li}^{+}:-3.89 \\
\mathrm{Na}^{+}:-4.11 \\
\mathrm{NH}_{4}^{+}:-2.79 \\
\mathrm{Ca}^{2+}:-4.97 \\
\mathrm{Mg}^{2+}:-6.83 \\
\mathrm{Ba}^{2+}:-4.98 \\
\mathrm{Sr}^{2+}:-4.01 \\
\end{array}$ & $\begin{array}{l}\log K_{\mathrm{Ca}^{2+}, B^{n+}}^{\text {pot }} \\
\mathrm{Li}^{+}:-4.20 \\
\mathrm{Mg}^{2+}:-5.60 \\
\mathrm{Na}^{+}:-3.20 \\
\mathrm{Ba}^{2+}:-4.98 \\
\mathrm{NH}_{4}^{+}:-4.20 \\
\mathrm{~K}^{+}:-4.20\end{array}$ & $\begin{array}{l}\log K_{N O_{2}^{3}, B^{n+}}^{p o t} \\
\mathrm{SO}_{4}^{2-}:-2.2 \\
\mathrm{I}^{-} \quad: 1.84 \\
\mathrm{Br}^{-} \quad:-1 \\
\mathrm{SCN}^{-}: 2.67 \\
\mathrm{Cl}^{-} \quad:-2.70 \\
\mathrm{~F}^{-} \quad:-3.30\end{array}$ \\
\hline Cevap zamanı & $<15 \mathrm{~s}$ & $<10 \mathrm{~s}$ & $<5 \mathrm{~s}$ \\
\hline Potansiyel değişimi & $44 \pm 2 \mathrm{mV}$ & $25 \pm 6 \mathrm{mV}$ & $41 \pm 7 \mathrm{mV}$ \\
\hline Sensör ömrü & 8 hafta & 8 hafta & 10 hafta \\
\hline
\end{tabular}

Tablo 3. Bitkilerin tükettikleri nitrat, potasyum ve kalsiyum miktarları

\begin{tabular}{lccc}
\hline Yetiştirilen ürün (Bir bitki yatağında) & Nitrat miktarı $(\mathrm{M})$ & Potasyum miktarı $(\mathrm{M})$ & Kalsiyum miktarı $(\mathrm{M})$ \\
\hline Yeşilbiber & $0.015 \pm 0.001$ & $0.03 \pm 0.002$ & $0.02 \pm 0.003$ \\
Patlıcan & $0.04 \pm 0.005$ & $0.07 \pm 0.01$ & $0.05 \pm 0.009$ \\
\hline
\end{tabular}

Her bir veri üç bağımsız ölçümün ortalama değeridir. Sonuçlar standart sapma şeklinde ifade edilmiştir.

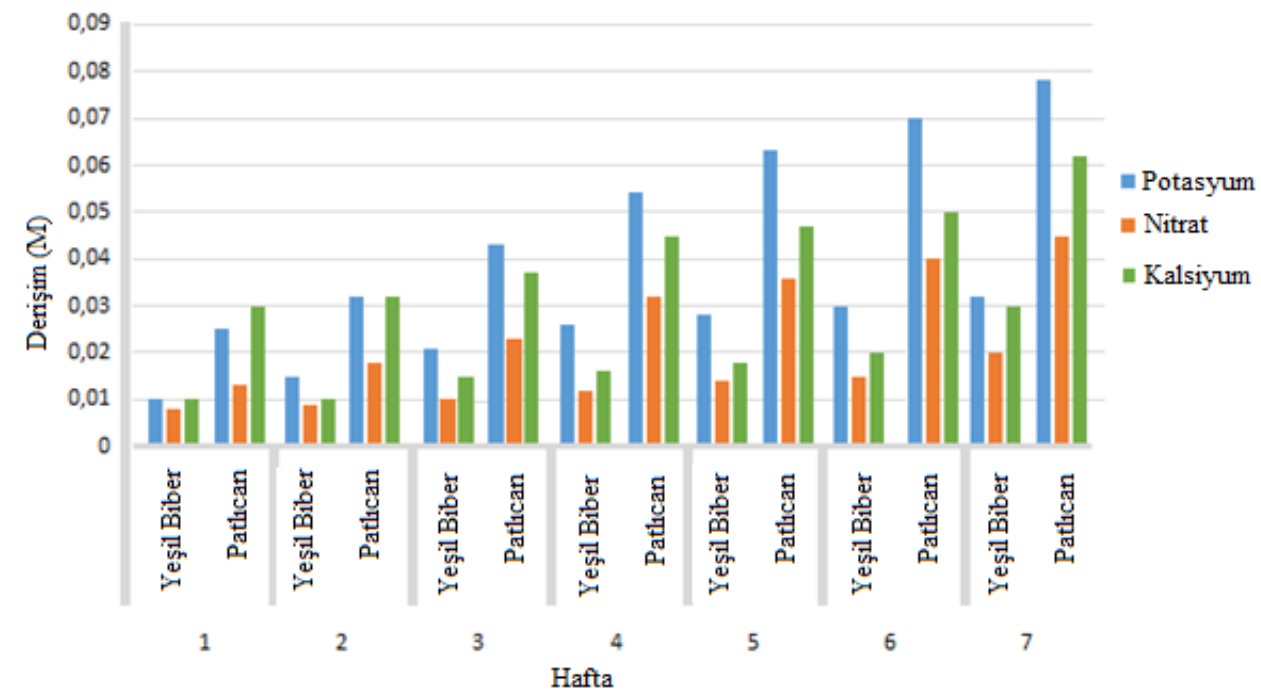

Şekil 2. Bitkilerin zamana bağlı besin elementleri tüketimi Deneysel koşullar; $25^{\circ} \mathrm{C}$ ve $\mathrm{pH} 7.0$ (Her bir veri noktası üç bağımsız deneyin ortalama değeridir) 
göstermektedir. Potasyum bitkinin yetişmesi için gerekli olan besin elementlerinden en bol miktarda bulunan katyondur (Gierth ve Mäser, 2007). Potasyum, azot ve fosfor ile beraber ürün verimini belirleyen önemli üç elementtir. $\mathrm{Bu}$ çalışmada, bitkilerin diğer besin elementlerine kıyasla daha fazla miktarda potasyuma ihtiyaç duyduğu belirlenmiştir. Şekil 2, biber ve patlican için tüketilen potasyum miktarını da göstermektedir. Patlıcanın yeşilbibere göre çok daha fazla miktarda potasyum tükettiği görülmüştür. Patlıcan tarafından kullanılan potasyum miktarında ilk 4 haftada hızlı, sonrasında azalan bir artış gözlenmiştir.

Hidrofonik besin çözeltisi içindeki kalsiyum iyon miktarı, bitki gelişimini önemli oranda etkileyen bir makro besin elementidir. Ortamda kalsiyumun eksikliği veya fazlalığı bitkilerin büyümesini olumsuz etkileyebilmektedir (NievesCordones ve ark., 2014). Yeşilbiber ve patlıcanın tükettiği kalsiyum miktarı yine Şekil 2'de görülmektedir. Sonuç olarak her iki bitkinin yapısal özellikleri karşılaştırıldığında yeşilbiber kısa bir zaman içerisinde patlıcana göre daha az besin miktarda besin elementlerinden kullanarak daha iyi ve hızlı bir büyüme göstermiştir.

\section{Sonuçlar}

$\mathrm{Bu}$ çalışma ile bitkiler ihtiyacı olan besin elementlerini topraktan almak yerine hazırlanmış besin solüsyonundan kontrollü bir şekilde daha kolay ve direkt olarak alabilecektirler. Besin ortamındaki (hidrofonik çözelti) bazı elementlerin $\left(\mathrm{NO}_{3}{ }^{-}, \mathrm{K}^{+}\right.$ve $\left.\mathrm{Ca}^{2+}\right)$ miktarlarının kontrolü ilk olarak bu çalışma ile PVC temelli iyon-seçici membran sensörler kullanılarak hızlı ve hassas olarak takip edilmiştir. PVC temelli iyon-seçici sensörler, hassasiyeti ve seçiciliğini sağlamak amacıyla nitrat için tetradodesilamonyum nitrat $\left(\mathrm{TDA}^{-\mathrm{NO}_{3}}{ }^{-}\right.$), potasyum için potasyum iyonofor ve kalsiyum için kalsiyum iyonofor kullanılarak hazırlanmıştır.

Bitki besin elementlerinin kontrollü bir şekilde bitkiye verilmesi ile daha sağlıklı ve hızlı yetişme sağlanabilir. İş gücüne gereksinimin azalması, üretime uygun olmayan yerlerde üretimin yap1labilmesi, bitkilerin beslenmesinin daha iyi kontrol edilebilmesi, bitki besleme ile ilgili harcama boyutunun azalması, kalite ve verimin daha yüksek olması, sudan tasarruf sağlanması, optimizasyona uygunluğu, tarımsal ilaç harcamalarının çok düşük olması, ekim nöbeti yapma zorunluluğu olmaması, üretimde devamlılığı sağlaması, birim alana daha fazla bitki dikilmesi ve erkencilik sağlaması; geliştirilecek sistemin diğer yaygın etkileri arasında olacaktır.
$\mathrm{Bu}$ takip ve izlenebilirlik ile bitki beslenme verimliliğine dair çok önemli bulgular edinildiğ $i$ düşünülmektedir. Bu sayede verim ve kalitenin önemli şekilde artacağı öngörülmektedir.

\section{Kaynaklar}

Anonim, 2016. Topraksız Tarım. http://www.bahce bitkileri.org/topraksiz-tarim-sunumu.html (Erişim tarihi: 02.10.2016).

Bailey, B.J., Haggett, B.G.D., Hunter, A., Albery, W.J., Svanberg, L.R., 1988. Monitoring nutrient film solutions using ion-selective electrodes. Journal of Agricultural Engineering Research, 40(2): 129-142.

Benoit, F., Ceustermans, N., 1995. Horticultural aspects of ecological soilless growing methods. Acta Horticulturae, 396: 11-24.

Blok, C., Kreij, C.D., Baas, R., Wever, G., 2008. Analytical methods used in soilless cultivation. In M. Raviv and JH. Lieth (Ed), Soilless Culture: Theory and Practice, Amsterdam, pp. 245-289.

Cloutier, G.R., Dixon, M.A., Arnold, K.E., 1997. Evaluation of sensor Technologies for automated control of nutrient solutions in life support systems using higher plants. In: Proceedings of the 6th European Symposium on Space Environmental Control Systems, Noordwijk, The Netherlands.

Çoldur, F., Andaç, M., Işıldak, İ., Saka, T., 2009. A micro-sized PVC membrane Li+-selective electrode without internal filling solution and its medical applications. Journal of Electroanalytical Chemistry, 626(1): 30-35.

Gierth, M., Mäser, P., 2007. Potassium transporters in plants - Involvement in $\mathrm{K}^{+}$acquisition, redistribution and homeostasis. FEBS Letters, 581(12): 2348-2356.

Gutierrez, M., Alegret, S., Caceres, R., Casadesus, J., Marfa, O., Del Valle, M., 2007. Application of a potentiometric electronic tongue to fertigation strategy in greenhouse cultivation. Computers and Electronics in Agriculture, 57(1): 12-22.

Işıldak, İ., Yolcu, M., Işıldak, Ö., Demirel, N., Topal, G., Hoşgören, H., 2004. All-solid-state PVC membrane $\mathrm{Ag}+$-selective electrodes based on diaza18-crown-6 compounds. Microchimica Acta, 144(1): $177-181$.

Jones, J.B., 2005. Hydroponics: A Practical Guide for the Soilless Grower. 2nd ed. CRC Press, Boca Raton, FL.

Kaplan, M., Sönmez, S., Tokmak, S., 1999. AntalyaKumluca yöresi kuyu sularının nitrat içerikleri. Turkish Journal of Agriculture and Forestry, 23: 309-313.

Meriç, M.K., Tüzel, İ.H., Tüzel, Y., Öztekin, G.B., 2011. Effects of nutrition systems and irrigation programs on tomato plants in soilless culture. Agricultural Water Management, 99(1): 19-25.

Nieves-Cordones, M., Aleman, F., Martinez, V., Rubio, F., 2014. $\mathrm{K}^{+}$uptake in plant roots. The systems involved, their regulation and parallels in other organisms. Journal of Plant Physiology, 171(9): 688-695. 
Pardossi, A., Tognoni, F., Incrocci, L., 2004. Mediterranean greenhouse technology. The World of Horticulture, 44: 28-34.

Raviv, M., Lieth, J.H., 2008. Soilless Culture: Theory and Practise. Elsevier, Amsterdam, The Netherlands, p. $343-424$.

Savvas, D., 2002. SW-Soil and water: Automated replenishment of recycled greenhouse effluents with individual nutrients in hydroponics by means of two alternative models. Biosystems Engineering, 83(2): 225-236.

Sönmez, İ., Kaplan, M., 2004. Demre yöresi seralarında toprak ve sulama sularının tuz içeriğinin belirlenmesi. Akdeniz Üniversitesi Ziraat Fakültesi Dergisi, 17(2): 155-160.

Van Os, E.A., Gieling, T.H., Ruijs, M.N.A., 2002. Equipment for hydroponic installations. In: D. Savvas and H. Passam, HC (Eds), Hydroponic Production of Vegetables and Ornamentals, Embryo Publications, Athens, Greece, pp. 103-141.

Vardar, G., Altıkatoğlu, M., Onat, D., Cemek, M, Işıldak, İ., 2015. Calcium, potassium and nitrate in plant nutrient solutions by measuring of with ionselective electrodes in hydroponic green house of some vegetables. Biotechnology and Applied Biochemistry, 62(5): 663-668. 